\title{
Advances in analytical separations
}

\author{
Uwe Karst • Martin Vogel
}

Published online: 13 May 2009

(C) Springer-Verlag 2009

This special issue of Analytical and Bioanalytical Chemistry contains a selection of 12 manuscripts originating from lectures and posters presented at the 27th International Symposium on Chromatography, held in Münster, Germany, from September 21 to 25, 2008. Both the conference and the articles impressively demonstrate that analytical separations are far from only being mature routine techniques with limited potential for innovation. Yes, these techniques are established tools in industry and academia owing to their high separation power and excellent performance/cost ratio. However, they also are one of the most important driving forces for innovation in many established and emerging areas of science.

The topics covered in these contributions range from the development of stationary phases to the development and application of complex hyphenated techniques. As probably the best example, liquid chromatography/mass spectrometry has strongly pushed liquid chromatography to new heights, and its application areas and significance in industry are still growing rapidly. It is therefore not surprising that it also plays a major role in this special issue. Two manuscripts on electrodriven analytical separation techniques complement the chromatography manuscripts for the analysis of ionic compounds.

We wish the readers interesting and rewarding reading of the articles in "Advances in Analytical Separation."

U. Karst $(\bowtie) \cdot$ M. Vogel

Institute of Inorganic and Analytical Chemistry,

University of Münster,

Corrensstr. 30,

48149 Münster, Germany

e-mail: uk@uni-muenster.de

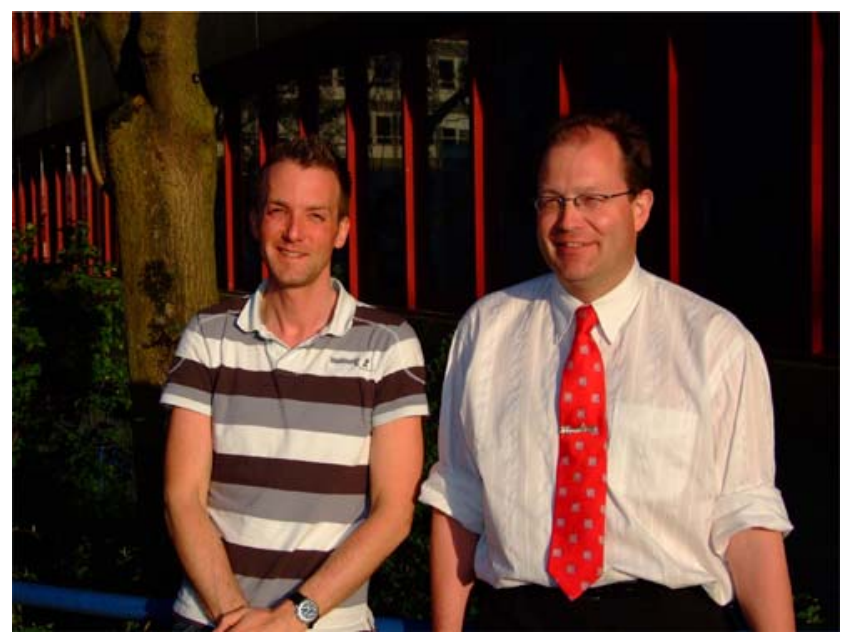

Uwe Karst (right) has held the Chair of Analytical Chemistry at the University of Münster since 2005. His research interests include hyphenated techniques and speciation analysis.

Martin Vogel (left) has had a faculty position at the University of Münster since 2006. His research interests include liquid chromatography/mass spectrometry and biochemical detection schemes for liquid chromatography. 